\title{
Walliser Ärzte lancieren ein Referendum für eine transparente, fachlich fundierte Gesundheitspolitik
}

\section{Monique Lehky Hagen}

Präsidentin des Referendumskomitees LeisReferendum
Korrespondenz:

Dr. med. Monique Lehky Hagen

FMH Innere Medizin

Postfach 596

CH-3900 Brig

info@leisreferendum.ch

www.leisreferendum.ch
Aufgrund der schweizweiten Einführung der Abrechnung nach Fallkostenpauschalen DRG ab 2012 müssen die entsprechenden kantonalen Gesetzgebungen angepasst werden.

Anlässlich der Revision des «Gesetzes über die Krankenanstalten- und Institutionen» (GKAI) im Wallis bot sich damit gleichzeitig die Gelegenheit, die Artikel in Bezug auf die Spitalplanung anzupassen, um gewisse Missstände zu verbessern. Leider hat der Walliser Grosse Rat weder die Stellungnahme der Walliser Ärztegesellschaft noch die Anliegen eines von über 200 Ärzten unterschriebenen Briefs berücksichtigt, so dass sich ein fast 40-köpfiges Ärztekomitee formiert hat, das nun ein Referendum gegen dieses revidierte Gesetz lanciert hat.

Im Kanton Wallis bestehen grosse sprachliche Probleme bei Hospitalisierung von nur grundversicherten Oberwalliser deutschsprachigen Patienten im Spital Sion, wo die spezialisierten Disziplinen zentralisiert wurden (z. B. Neurochirurgie, Herz- und Thoraxchirurgie, invasive Kardiologie, Radioonkologie usw.). Eine adäquate Kommunikation mit dem Ärzte- und Pflegepersonal ist nicht gewährleistet, und es wurden diesbezüglich in den letzten 10 Jahren keine nennenswerten Initiativen unternommen, um eine Verbesserung der Deutschsprachigkeit in einem so wichtigen Sektor zu erreichen. Dies erstaunt umso mehr, als es nun einen nationalen Telefondolmetschdienst für den Gesundheitsbereich in 12 Nicht-Landessprachen gibt! Dies sollte die Bedeutung dieses Problems mehr als verdeutlichen ...

Im Bereich der Spitalplanung hat die Ärzteschaft seit Jahren kaum mehr ein Wort zu sagen und wird als rein ausführendes Organ angesehen. Dies wird verdeutlicht durch die Tatsache, dass die klar formulierten Forderungen der Ärzteschaft kommentarlos ignoriert werden können, und auch ein offener Brief an den Staatsrat bisher unbeantwortet geblieben ist.

Aufgrund diverser Skandale im Walliser Spitalbereich 2010 wurde vom Grossrat gegen den Willen des Staatsrats ein Audit des Walliser Spitalsystems (RSVGNW, neu «Spital Wallis») in Auftrag gegeben, das 7 Monate dauerte und über 1 Million Franken Steuergelder gekostet hat.
In diesem Audit wird klar auf relevante Probleme in der administrativen Struktur und der Personalführungsstruktur des RSV hingewiesen. Diese Probleme können nur durch Änderungen gewisser Gesetzesartikel, die im GKAI festgelegt sind, behoben werden. Der Grossrat hatte die Verschiebung der definitiven Abstimmung über das Gesetz im März 2011 entgegen der Forderung der Ärzte abgelehnt, im Wissen, dass die Resultate dieses grossen Audits kurz vor der Veröffentlichung standen, die 10 Tage später erfolgte. Ebenfalls in diesem Zeitraum wurde der Geschäftsführungskommissionsbericht (GPK) vorgelegt, der ähnliche Schlussforderungen wie das Audit formulierte.

Nach Gesprächen mit den verschiedenen involvierten Parteien, inklusive den Präsidenten der Oberwalliser Parteien (resp. deren Vertreter), sind wir zum Schluss gekommen, dass wir aus ethischen und standespolitischen Gründen verpflichtet sind, ein Referendum gegen dieses Gesetz zu ergreifen.

Unsere Forderungen sind:

- Verankerung der Zweisprachigkeit für zentralisierte und delegierte Disziplinen;

- Adäquate Berücksichtigung und Integration sachlicher Argumente medizinischer Fachpersonen in die Gesetzgebung;

- Adäquate Berücksichtigung und Integration der Resultate des Audits und GPK-Berichts ins GKAI.

Wir fühlen uns verpflichtet, uns für eine optimale Gesundheitsversorgung unserer Walliser Bevölkerung einzusetzen und in diesem Sinne Transparenz und Sachpolitik in der Gesundheitspolitik zu fordern.

Die aktuelle Situation sollte uns alle aufhorchen lassen, an welchem Tiefpunkt wir standespolitisch angelangt sind.

Wir hoffen auf eine grosse Mobilisation der Ärzteschaft und der Bevölkerung, damit wir uns in so wichtigen Belangen Gehör und Mitwirkungsrecht verschaffen können, um für das Wohl unserer Patienten und eine optimale Zusammenarbeit der verschiedenen Player im Gesundheitssystem eintreten zu können.

Mehr Informationen finden sich unter www. leisreferendum.ch 